\title{
Role of Circulating Vascular Cell Adhesion Protein-1 as a Biomarker in Non-Alcoholic Fatty Liver Disease
}

\author{
Ahmed Samir Abo Halima*1, Mohamed Abd EL Moghny Mostafa ${ }^{1}$, Ahmed Ali El Ray², \\ Yasser Mohamed Ali Abdelhady Alsaid ${ }^{2}$, Khaled Raafat ${ }^{1}$ \\ ${ }^{1}$ Internal Medicine and Gastroenterology Department, Faculty of Medicine, Ain Shams University, Egypt \\ ${ }^{2}$ Internal Medicine and Gastroenterology Department, Faculty of Medicine, October 6 University, Egypt \\ *Corresponding Author: Ahmed Samir Abo Halima, Mobile: (+20)1111194743, E-mail: dr.abohalima@hotmail.com
}

\begin{abstract}
Background: As a result of the obesity pandemic, non-alcoholic fatty liver disease (NAFLD) has become the most common cause of chronic liver disease worldwide. NAFLD is the hepatic manifestation of obesity and a precursor of and independent risk factor for type 2 diabetes.

Objective: The aim of the current work was to assess the level of vascular cell adhesion molecule 1 (VCAM-1) as noninvasive diagnostic tools for diagnosis of NAFLD degree of fibrosis.

Patients and Methods: This Case-Control clinical study included a total of 60 subjects, 30 patients with fatty liver disease, FLD (Group A) and 30 healthy subjects as a control (group B), attending at Internal Medicine and Hepatology Outpatient Clinic, Ain Shams University Hospitals. Group A was further subdivided into NAFLD subgroup (15 patients) and non- alcoholic steatohepatitis (NASH) subgroup (15 patients)

Results: In the present study, the mean VCAM-1 was $2.392 \pm 0.3$ in NAFLD group, $9.893 \pm 2.3$ in NASH group and $1.983 \pm 0.3$ in control group with high statistically significant increase in NASH followed by NAFLD than in control group. Regarding to ROC curve, VCAM-1 had excellent Diagnostic performance with an AUC of 0.994. A best cut-off criterion of VCAM-1 > 7.7 ng/mL could discriminate between patients with NASH from control group with a sensitivity of $95 \%$ and specificity of $100 \%$ In our study, there was no significant correlation in between VCAM-1 and age, AST, ALT. In the present study, the significant predictors of bad outcome in patients with NAFLD and NASH were higher VCAM-1, GGT and AST levels.
\end{abstract}

Conclusion: It could be concluded that the significant predictors of bad outcome in patients with NAFLD and NASH were higher VCAM-1 level, GGT and higher AST levels.

Keywords: Circulating Vascular cell adhesion protein - 1, Non-alcoholic Fatty Liver Disease

\section{INTRODUCTION}

As a result of the obesity pandemic, nonalcoholic fatty liver disease (NAFLD) has become the most common cause of chronic liver disease worldwide. NAFLD is the hepatic manifestation of obesity and considered as independent risk factor for type 2 diabetes (1).

NAFLD comprises a spectrum of disease that ranges from hepatocellular steatosis without necroinflammation (NAFL) to non- alcoholic steatohepatitis (NASH), fibrosis, cirrhosis, and even hepatocellular carcinoma. In addition, NAFLD is an independent risk factor for cardiovascular disease. Recent studies showed an increased cardiovascular mortality in NAFLD patients ${ }^{(2)}$.

The global prevalence of NAFLD and NASH is around $25 \%$ and $3 \%$, respectively, although this rises to an estimated $90 \%$ and $25 \%$, respectively, in severely obese patients ${ }^{(3)}$.

Liver biopsy is still considered as the gold standard for the diagnosis of NASH and the assessment of disease activity and fibrosis, although it has important disadvantages such as its high cost, invasive nature, and the risk of sampling error ${ }^{(4)}$.

This has inspired the search for non-invasive disease markers, including both serum biomarkers and imaging methods. Nevertheless, there are currently no non-invasive markers that can adequately distinguish NAFLD from NASH ${ }^{(5)}$.

In this way, the role of circulating biomarkers related to endothelial dysfunction and the severity of underlying liver disease need to be investigated. It is suggested that there is a role of VCAM-1 stimulating adhesion of lymphocyte and monocytes to the surface of the vascular endothelium. VCAM-1 is not investigated thoroughly in different stages of liver diseases, such as NAFLD ${ }^{(6)}$.

The aim of the present study was to assess the level of vascular cell adhesion molecule-1 (VCAM-1) as non-invasive diagnostic tools for diagnosis of NAFLD degree of fibrosis

\section{PATIENTS AND METHODS}

This Case-Control clinical study included a total of 60 subjects, 30 patients with fatty liver disease (Group A) and 30 healthy subjects as a control (group B), attending at Internal Medicine and Hepatology Outpatient Clinic, Ain Shams University Hospitals.

Patients of Group A was further subdivided into NAFLD subgroup (15 patients) and non- alcoholic steatohepatitis (NASH) subgroup (15 patients)

Inclusion Criteria: Egyptian outpatient adults over 18 years, patients with elevated AST and ALT and 
hepatomegaly with increased echogenicity in abdominal ultrasonography.

Exclusion Criteria: Patients with insufficient clinical and/or laboratory data. Remove, patients with other diagnosed chronic liver diseases as viral hepatitis, metabolic and genetic disorders as Hemochromatosis, Wilson disease, autoimmune hepatitis, and drug induced liver disease. Patients with other causes of elevated liver enzymes apart from NASH, patients with recent infection and those who refused to be entitled in the study remove.

\section{Ethical Considerations:}

All procedures in this study were follow Ain Shams University Ethical committee regulations and patient consent was taken from all subjects, all included patients had an informed written consent after explanation the aim of the study, any unexpected risks were cleared to the Ethics Committee and patients on time and privacy of participants as follows: We used code numbers for every patient as a symbol to the name and address that was kept in special file, result of the research was used for scientific aim only and not for any other aims and we hid the patients' names when we used this research. This work has been carried out in accordance with The Code of Ethics of the World Medical Association (Declaration of Helsinki) for studies involving humans.

\section{Pre-operative preparation:}

Full History Taking: Detailed history was taken regarding the onset of the disease, the presenting, any previous treatments, and their types whether surgical or medical, any previous laboratory investigations (urine or blood analysis), and any previous imaging procedures. Demographic information was obtained including age, gender, address, phone number, smoking status, alcohol consumption, presence of family history, skin color, etiology and presence of ascites, the patients were also asked about family history. Also detailed history was taken about lifestyle, duration of disease and patients were asked about past medical history of any previous disease, trauma, surgery and specific treatment received too long.

Thorough Clinical Examination: General physical examination was also carried out; systolic and diastolic blood pressure was measured twice every $5 \mathrm{~min}$ on the right arm after $5 \mathrm{~min}$ of rest with a sphygmomanometer. The mean of the two readings was used in data analysis, vital signs (heart rate, pulse, temperature, and respiratory rate), all patients were evaluated for systemic diseases or precipitating causes and general examination: Cardiac, chest and neurological examination

Abdominal examination: Abdominal contour, inspection, superficial palpation for liver, spleen \& loin, percussion, and auscultation Remove
Anthropometric Examinations: Body weight is measured to an accuracy of $0.1 \mathrm{~kg}$ in light indoor clothing without shoes, and height is measured using a wall-mounted stadiometer. Body mass index (BMI) is calculated as body weight $/$ height $^{2}\left(\mathrm{~kg} / \mathrm{m}^{2}\right)$. Waist circumference was measured at the umbilicus, anthropometric examinations, including waist circumference, and hip circumference were performed according to standard procedures and waist circumflex (WC) was measured midway between the uppermost border of the iliac crest and the lower border of the costal margin.

Full Routine Laboratory Investigations: liver function tests including: (ALT, AST, $\gamma$-glutamyl transpeptidase (GGT), total bilirubin, direct bilirubin and albumin were done by kinetic method. Complete blood count (CBC), hepatitis markers for screening (hepatitis B surface antigen (HBsAg) and hepatitis C antibody ( $\mathrm{HCV} \mathrm{Ab}$ ), fasting plasma glucose, $\mathrm{HbA1c}$, serum creatinine, urea, $\mathrm{Na}$ and $\mathrm{K}$, lipid profile (triglycerides, total cholesterol, HDL, LDL) and abdominal ultrasonography were also done.

Ultrasound is the most widely available modality for initial evaluation of suspected hepatic steatosis, usually after detection of abnormal liver function tests. It is noninvasive, easy to perform and inexpensive and does not expose the patient to ionizing radiation.

Determination of serum VCAM-1 levels was done by the enzyme-linked immunosorbent assay (ELISA) Kit for (Chongqing Biospes, China).

\section{Statistical Analysis:}

Collected data sheets was entered to excel sheet, then all data were exported to SPSS (Statistical Package for Social Sciences); SPSS Inc., Chicago, IL, USA Program. Data were analyzed using SPSS version 24; Data were presented as numbers and percentage, mean \pm standard deviation, median [25th, 75th percentiles] or frequency (percent). A univariable analysis was performed to assess differences between the 2 diagnosis groups (Healthy Controls, and NAFLD); analysis of variance (ANOVA) or the non-parametric KruskalWallis tests was used to assess differences in continuous or ordinal variables and Pearson's chi-square tests was used for categorical factors. VCAM-1 level comparison between both groups was carried out using independent samples t-test. ROC CURVE test was used to assess the sensitivity and specificity of the VCAM-1 level as a Biomarker for detection of NAFLD. P-value less than 0.05 was considered statistically significant.

\section{RESULTS}

Table 1 shows that the mean age in FLD group was $58.46 \pm 4.37 \mathrm{y}$ while in control group was $59.25 \pm 7.83$ y with no significant difference between them. There was male predominance in both groups and there were no significant statistical differences regarding diabetes, smoking and HTN. 
Table (1): Clinical characteristics of the studied patients

\begin{tabular}{|l|c|c|c|c|}
\hline \multicolumn{1}{|c|}{ Items } & $\begin{array}{c}\text { FLD group }(\mathbf{n}=\mathbf{3 0}) \\
\text { No }(\%)\end{array}$ & $\begin{array}{c}\text { CONTROL group } \\
(\mathbf{n}=\mathbf{3 0}) \\
\text { No(\%) }\end{array}$ & $\chi^{\mathbf{2}}$ & p-value \\
\hline $\begin{array}{l}\text { Age (years): } \\
\text { Mean } \pm \text { SD }\end{array}$ & $58.46 \pm 4.37$ & $59.25 \pm 7.83$ & $\mathrm{t}=0.32$ & 0.778 \\
\hline $\begin{array}{l}\text { Sex: } \\
\text { Males }\end{array}$ & $24(80)$ & $19(63.3)$ & & \\
Females & $6(20)$ & $11(36.7)$ & 2.051 & $\mathbf{0 . 1 5 2}$ \\
\hline Diabetes Mellitus & $8(26.7)$ & $6(20)$ & 0.571 & $\mathbf{0 . 4 4 9}$ \\
\hline Smoking & $12(40)$ & $9(30)$ & 0.857 & $\mathbf{0 . 3 5 4}$ \\
\hline HTN & $8(26.7)$ & $9(30)$ & 0.117 & $\mathbf{0 . 7 3 1}$ \\
\hline
\end{tabular}

In table 2, the mean VCAM-1 was 2.392 0.3 in NAFLD group, 9.893 \pm 2.3 in NASH group and $1.983 \pm 0.3$ in control group with high statistically significant difference in between.

Table (2): Comparison between the different studied groups according to VCAM-1

\begin{tabular}{|c|c|c|c|c|c|}
\hline $\begin{array}{c}\text { VCAM-1. } \\
(\mathbf{n g} / \mathbf{m l})\end{array}$ & $\begin{array}{c}\text { NAFLD group } \\
(\mathbf{n}=\mathbf{1 5})\end{array}$ & $\begin{array}{c}\text { NASH group } \\
(\mathbf{n}=\mathbf{1 5})\end{array}$ & $\begin{array}{c}\text { CONTROL } \\
\text { group }(\mathbf{n}=\mathbf{3 0})\end{array}$ & $\mathbf{F}$ & $\mathbf{p}$ \\
\hline Mean \pm SD & $2.392 \pm 0.3$ & $9.893 \pm 2.3$ & $1.983 \pm 0.3$ & $97.735^{*}$ & $0.003^{*}$ \\
\hline
\end{tabular}

As in table 3, VCAM-1 had excellent Diagnostic performance with an AUC of 0.747. A best cut-off criterion of VCAM-1 $>2.3 \mathrm{ng} / \mathrm{mL}$ could discriminate between patients with NAFLD from control group with a sensitivity of 55\% and specificity of 100\%. VCAM-1 had excellent Diagnostic performance with an AUC of $\mathbf{0 . 9 9 4}$. A best cut-off criterion of VCAM-1 $>7.7 \mathrm{ng} / \mathrm{mL}$ could discriminate between patients with NASH from control group with a sensitivity of $95 \%$ and specificity of $100 \%$

Table (3): Sensitivity, specificity and cutoff value of VCAM-1 to detect NAFLD group and NASH group

\begin{tabular}{|c|c|c|c|c|c|c|}
\hline & Cut off point & AUC & Sensitivity & Specificity & +PV & -PV \\
\hline NAFLD group & $>2.3$ & 0.747 & 55.00 & 100.00 & 100.0 & 52.6 \\
\hline NASH group & $>7.7$ & $\mathbf{0 . 9 9 4}$ & $\mathbf{9 5 . 0 0}$ & $\mathbf{1 0 0 . 0 0}$ & $\mathbf{1 0 0 . 0}$ & $\mathbf{9 5 . 2}$ \\
\hline
\end{tabular}

$\mathbf{A U C}=$ area under curve, $\mathbf{P P V}=$ positive predictive value, $\mathbf{N P V}=$ negative predictive value .

Table 4 shows that, the mean FIB- 4 score was $1.13 \pm 0.423$ in NFLD group, $1.63 \pm 0.750$ in NASH group and $0.13 \pm 0.03$ in control group with a significant difference in between them regarding to NAFLD fibrosis score.

Table (4): Comparison between the two studied groups according to FIB-4 score

\begin{tabular}{|c|c|c|c|c|c|}
\hline FIB-4 score & $\begin{array}{c}\text { NAFLD group } \\
(\mathbf{n}=\mathbf{1 5})\end{array}$ & $\begin{array}{c}\text { NASH group } \\
(\mathbf{n}=\mathbf{1 5})\end{array}$ & $\begin{array}{c}\text { CONTROL group } \\
(\mathbf{n}=\mathbf{3 0})\end{array}$ & \multirow{2}{*}{$\mathbf{x}^{\mathbf{2}}$} & $\mathbf{p}$ \\
\hline Mean \pm SD. & $1.13 \pm 0.423$ & $1.63 \pm 0.750$ & $0.13 \pm 0.03$ & \multirow{2}{*}{8.272} & \multirow{2}{*}{$0.021 *$} \\
\hline Median (IQR) & 1.14 & 1.633 & 0.13 & & 0.13 \\
\hline
\end{tabular}

$\mathrm{p}: \mathrm{p}$ value for comparing between the studied groups

*: Statistically significant at $\mathrm{p} \leq 0.05$

In table 5, there was a significant difference in between NAFL, NASH and control group regarding to APRI score while no significant difference in between them regarding to NAFLD fibrosis score.

Table (5): Comparison between the two studied groups according to APRI score

\begin{tabular}{|c|c|c|c|c|c|}
\hline & $\begin{array}{c}\text { NAFLD } \\
\text { group }(n=15)\end{array}$ & $\begin{array}{c}\text { NASH group } \\
(n=15)\end{array}$ & $\begin{array}{c}\text { CONTROL } \\
\operatorname{group}(n=30)\end{array}$ & $\mathbf{x}^{2}$ & $\mathbf{p}$ \\
\hline APRI score & & & & \multirow{3}{*}{6.130} & \multirow{3}{*}{$0.044 *$} \\
\hline Mean \pm SD. & $\mathbf{0 . 8 1 6} \pm 0.416$ & $\mathbf{0 . 8 8 4} \pm 0.154$ & $\mathbf{0 . 3 6 2} \pm 0.04$ & & \\
\hline Median (IQR) & 0.845 & 0.694 & 0.375 & & \\
\hline $\begin{array}{c}\text { NAFLD fibrosis score } \\
\text { Mean } \pm \text { SD. } \\
\text { Median (IQR) }\end{array}$ & $\begin{array}{c}-0.891 \pm 0.354 \\
-0.633\end{array}$ & $\begin{array}{c}-0.998 \pm 0.315 \\
-0.746\end{array}$ & $\begin{array}{c}-2.053 \pm 0.555 \\
-1.955\end{array}$ & 0.829 & 0.660 \\
\hline
\end{tabular}

$\mathrm{p}$ : $\mathrm{p}$ value for comparing between the studied groups

*: Statistically significant at $\mathrm{p} \leq 0.05$ 
Table 6 shows that there was a significant correlation in between VCAM-1 and ALT, AST, albumin, bilirubin, GGT, HDL, LDL, HbA1C, and fibrosis-4 (FIB-4) in NASH group

Table (6): Correlation between VCAM-1and FIB-4, APRI score and NAFLD score in the NASH group

\begin{tabular}{|c|c|c|}
\hline \multirow{2}{*}{ Parameter } & \multicolumn{2}{|c|}{ VCAM-1 } \\
\cline { 2 - 3 } & $\mathbf{R}$ & $\mathbf{P}$ \\
\hline FIB-4 & $\mathbf{0 . 7 2 3}$ & $\mathbf{0 . 0 0 0} *$ \\
\hline APRI score & $\mathbf{0 . 0 3 8}$ & $\mathbf{0 . 8 1 5}$ \\
\hline NAFLD fibrosis score & $\mathbf{0 . 3 3 3}$ & $\mathbf{0 . 1 5 2}$ \\
\hline
\end{tabular}

Table 7 shows that there was a significant correlation in between VCAM-1 and GGT in NAFLD group.

Table (7): Correlation between VCAM-1 and FIB-4, APRI score and NAFLD score different parameters in NAFLD group

\begin{tabular}{|c|c|c|}
\hline \multirow{2}{*}{ Parameter } & \multicolumn{2}{|c|}{ VCAM-1 } \\
\cline { 2 - 3 } & $\mathbf{R}$ & $\mathbf{P}$ \\
\hline FIB-4 & $\mathbf{0 . 2 0 2}$ & $\mathbf{0 . 3 6 6}$ \\
\hline APRI score & $\mathbf{0 . 0 1 9}$ & $\mathbf{0 . 9 2 0}$ \\
\hline NAFLD fibrosis score & $\mathbf{0 . 3 0 7}$ & $\mathbf{0 . 0 9 9}$ \\
\hline
\end{tabular}

In table 8 shows that, the significant predictors of bad outcome in patients with NAFL and NASH were higher VCAM1 level, GGT and higher AST levels.

Table (8): Binary logistic regression analysis for relevant predictors of the outcome of patients with NASH and NAFLD

\begin{tabular}{|c|c|c|c|c|c|}
\hline \multirow{2}{*}{ Predictors } & \multirow{2}{*}{ B } & \multirow{2}{*}{ p-value } & \multirow{2}{*}{ OR } & \multicolumn{2}{|c|}{ 95\% CI } \\
\cline { 5 - 6 } & & & & Lower limit & Upper limit \\
\hline VCAM-1 (ng/ml) & $\mathbf{0 . 1 6 1}$ & $\mathbf{0 . 0 2 6}$ & $\mathbf{0 . 8 5 1}$ & $\mathbf{0 . 7 3 9}$ & $\mathbf{0 . 9 8 1}$ \\
\hline Albumin (g/dL) & $\mathbf{0 . 0 2 9}$ & $\mathbf{0 . 2 6 9}$ & $\mathbf{1 . 0 2 9}$ & $\mathbf{0 . 9 7 8}$ & $\mathbf{1 . 0 8 4}$ \\
\hline FIB-4 & $\mathbf{0 . 3 4 1}$ & $\mathbf{0 . 0 9 2}$ & $\mathbf{1 . 4 0 7}$ & $\mathbf{0 . 9 4 6}$ & $\mathbf{2 . 0 9 3}$ \\
\hline GGT (IU/L) & $\mathbf{0 . 0 1 9}$ & $\mathbf{0 . 0 0 7}$ & $\mathbf{1 . 0 1 9}$ & $\mathbf{0 . 9 9 9}$ & $\mathbf{1 . 0 4 1}$ \\
\hline ALT (U/L) & 1.839 & $\mathbf{0 . 0 5 7}$ & $\mathbf{6 . 2 9 0}$ & $\mathbf{0 . 9 4 4}$ & $\mathbf{4 1 . 8 9 5}$ \\
\hline AST (U/L) & $\mathbf{0 . 0 1 9}$ & $\mathbf{0 . 0 0 6 7}$ & $\mathbf{1 . 0 1 9}$ & $\mathbf{0 . 9 9 9}$ & $\mathbf{1 . 0 4 1}$ \\
\hline
\end{tabular}

\section{DISCUSSION}

In the current study, there was male predominance in both groups (80\% in NAFLD, $63.3 \%$ IN control) which agree with Lee et al. ${ }^{(7)}$ who found male predominance $(52.1 \%)$. Also, our results agreed with the study by Kraemer $\boldsymbol{e t}$ al. ${ }^{(8)}$ which involved 322 patients (98 women and 224 men).

In the present study, there was non-significant differences between NAFLD and control groups regarding to age, hypertension and diabetes which in line with study of Weston et al. ${ }^{(9)}$ who had the same results.

In the current study, there was a significant elevation of triglyceride in NASH followed by NAFLD then control which agree with Weston et al. (9) who found that Cholesterol levels were similar in each cohort, but those with NAFLD had higher triglyceride levels.

In the present study, there was non-significant difference in between NASH, NAFLD and control regarding to HDL but a significant difference regarding to LDL which partially agree with study of Lefere et $\boldsymbol{a l} .{ }^{(6)}$ who stated that there was non- significant difference in between NASH, NAFLD and control regarding to HDL and LDL

As regard to fasting plasma glucose, there was highly statistically significant increase in NASH followed by NAFLD and then control groups. In a meta-analysis by Dai et al. ${ }^{\left({ }^{(10)}\right.}$ of 24 studies, the pooled prevalence of NAFLD in T2DM patients was almost $60 \%$. In another more recent by Younossi et al. (11) meta-analysis, the global prevalence of NAFLD among T2DM patients was $55.5 \%$. Similarly, a metaanalysis by Atan et al. ${ }^{(\mathbf{1 2})}$ that included 17 studies with T2DM patients found the prevalence of NAFLD to be $54 \%$.

In the current study, there was a statistically significant differences in between NASH, NAFLD and control groups regarding to AST and ALT which partially agree with Lefere $\boldsymbol{e} \boldsymbol{a l}$. $^{\left({ }^{(6)}\right.}$ who stated that there was non-significant difference in between NASH, NAFLD and control regarding to ALT and but a significant difference in between NASH, NAFLD and control regarding to AST

In the present study, the mean VCAM-1 was $2.392 \pm 0.3$ in NAFLD group, $9.893 \pm 2.3$ in NASH group and $1.983 \pm 0.3$ in control group with high 
statistically significant increase in NASH followed by NAFLD than in control group.

That agree with Shepherd et al. ${ }^{(13)}$ who stated that profound increase in hepatic expression of VAP-1 in NASH and other cirrhotic disease which also in agreement with previous evidence ${ }^{(9,14)}$.

In the current study, there was a significant positive correlation in between VCAM-1 and BMI in both NAFLD and NASH groups Similar results have been reported in vivo where benzylamine increases adipose tissue fat deposition in diabetic rats ${ }^{(\mathbf{1 5})}$, and transgenic mice overexpressing VAP-1/SSAO supplemented with methylamine have increased BMI and abdominal fat pad weight ${ }^{(\mathbf{1 6})}$.

In this study, there was a significant positive correlation in between VCAM-1 and bilirubin which agree with Keshavan et al. ${ }^{(17)}$ who stated that Consistent with the inhibitory function of bilirubin on VCAM-1-dependent leukocyte transmigration in vitro, bilirubin blocks VCAM-1-dependent inflammation by blocking VCAM-1 signaling. In the present study, there was a significant positive correlation in between VCAM-1 and GGT in both NAFLD and NASH groups Kraemer et al. ${ }^{\left({ }^{(8)}\right.}$ also found a significant correlation between VCAM-1 and GGT.

According to $\mathrm{HbA1c}$, there was a significant positive correlation in between VCAM-1 and HbA1c. Increased SVAP-1 expression and modification of GLUT expression were concomitantly observed with changes in insulin levels and HbA1c, which may represent an attempt to regulate blood glucose levels (14). However, this chronic ectopic activity may subsequently promote vascular complications, influencing AGE formation, atherosclerosis, glomerulosclerosis and hypertension ${ }^{(16)}$.

In our study, there was no significant correlation in between VCAM-1 and age, AST, ALT which coincide with Lefere et al. ${ }^{(6)}$ who stated that no significant correlation in between VCAM-1 and age, BMI, HOMA-IR, AST, ALT or cholesterol

In the present study, the significant predictors of bad outcome in patients with NAFLD and NASH were higher VCAM-1 level, GGT and higher AST levels. Weston et al. ${ }^{\left({ }^{9}\right)}$ stated that circulating SVAP-1 correlates with disease severity and is predictive of NASH and the presence of significant fibrosis on biopsy in NAFLD. In the study done by Kraemer $\boldsymbol{e} t$ al. ${ }^{(8)}$ VAP-1 concentration was the second strongest variable associated with liver stiffness after GGT.

\section{CONCLUSION}

It could be concluded that the significant predictors of bad outcome in patients with NAFLD and NASH were higher VCAM-1 level, GGT and higher AST levels.

Financial support and sponsorship: Nil. Conflict of interest: Nil.

\section{REFERENCES}

1. Lonardo A, Ballestri S, Marchesini G et al. (2015): Nonalcoholic fatty liver disease: a precursor of the metabolic syndrome. Dig Liver Dis., 47: 181-190.

2. Angulo P, Kleiner D, Dam-Larsen S et al. (2015): Liver fibrosis, but no other histologic features, is associated with long-term outcomes of patients with nonalcoholic fatty liver disease. Gastroenterology, 149: 389-397.e10.

3. Younossi Z, Koenig A, Abdelatif D et al. (2016): Global epidemiology of nonalcoholic fatty liver disease-meta-analytic assessment of prevalence, incidence, and outcomes. Hepatology, 64: 73-84

4. European Association for the Study of the Liver, European Association for the Study of Diabetes, European Association for the Study of Obesity (2016): EASL-EASDEASO Clinical Practice Guidelines for the management of non-alcoholic fatty liver disease. J Hepatol., 64: 1388-1402.

5. Machado M, Cortez-Pinto H (2013): Non-invasive diagnosis of non- alcoholic fatty liver disease. A critical appraisal. J Hepatol., 58: 1007-1019.

6. Lefere S, Van de Velde F, Devisscher L et al. (2017): Serum vascular cell adhesion molecule-1 predicts significant liver fibrosis in non-alcoholic fatty liver disease. International Journal of Obesity, 41: 1207-1213.

7. Lee J, Park Y, Yun J et al. (2020): The association between nonalcoholic fatty liver disease and esophageal, stomach, or colorectal cancer: National population-based cohort study. PLoS One, 15(1):e0226351.

8. Kraemer M, Krawczyk M, Noor F et al. (2019): Increased circulating VAP-1 levels are associated with liver fibrosis in chronic hepatitis C infection. Journal of Clinical Medicine, 8: 103107.

9. Weston C, Shepherd E, Claridge L et al. (2015): Vascular adhesion protein-1 promotes liver inflammation and drives hepatic fibrosis. J Clin Invest., 125(2): 501-520.

10. Dai W, Ye L, Liu A et al. (2017): Prevalence of nonalcoholic fatty liver disease in patients with type 2 diabetes mellitus: a metaanalysis. Medicine, 96: 8179-83.

11. Younossi Z, Golabi P, de Avila L et al. (2019): The global epidemiology of NAFLD and NASH in patients with type 2 diabetes: a systematic review and meta-analysis. J Hepatol., 71: 793-801.

12. Atan N, Koushki M, Motedayen M et al. (2017): Type 2 diabetes mellitus and non-alcoholic fatty liver disease: a systematic review and meta-analysis. Gastroenterol Hepatol Bed Bench, 10: 1-7.

13. Shepherd E, Karim S, Newsome $P$ et al. (2020): Inhibition of vascular adhesion protein-1 modifies hepatic steatosis in vitro and in vivo. World J Hepatol., 12: 931-48.

14. Karim S, Liaskou E, Fear J et al. (2014): Dysregulated hepatic expression of glucose transporters in chronic disease: contribution of semicarbazide-sensitive amine oxidase to hepatic glucose uptake. Am J Physiol Gastrointest Liver Physiol., 307(12): 11801190.

15. Marti L, Abella A, Carpéné C et al. (2001): Combined treatment with benzylamine and low dosages of vanadate enhances glucose tolerance and reduces hyperglycemia in streptozotocin-induced diabetic rats. Diabetes, 50(9): 2061-2068.

16. Stolen M, Madanat C, Marti R et al. (2004): Semicarbazidesensitive amine oxidase overexpression has dual consequences: insulin mimicry and diabetes-like complications. FASEB J., 18: 702-704.

17. Keshavan P, Deem T, Schwemberger S et al. (2005): Unconjugated bilirubin inhibits VCAM-1-mediated transendothelial leukocyte migration. J Immunol., 174: 37093718. 